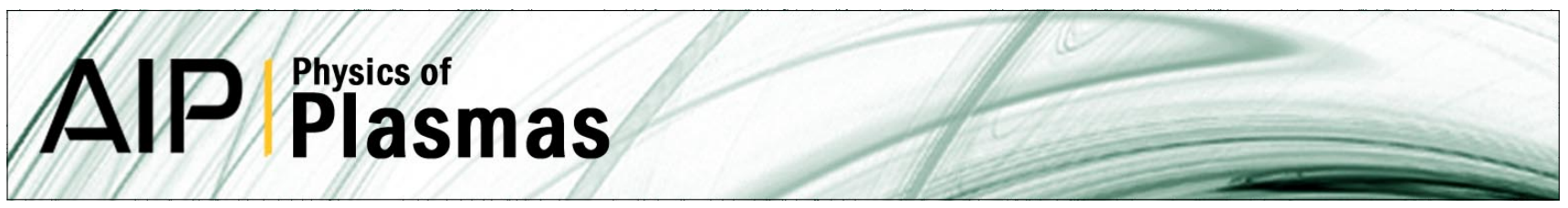

\title{
Intermittent fluctuations in the Alcator C-Mod scrape-off layer
}

O. E. Garcia, S. M. Fritzner, R. Kube, I. Cziegler, B. LaBombard et al.

Citation: Phys. Plasmas 20, 055901 (2013); doi: 10.1063/1.4802942

View online: http://dx.doi.org/10.1063/1.4802942

View Table of Contents: http://pop.aip.org/resource/1/PHPAEN/v20/i5

Published by the AIP Publishing LLC.

\section{Additional information on Phys. Plasmas}

Journal Homepage: http://pop.aip.org/

Journal Information: http://pop.aip.org/about/about_the_journal

Top downloads: http://pop.aip.org/features/most_downloaded

Information for Authors: http://pop.aip.org/authors

\section{ADVERTISEMENT}

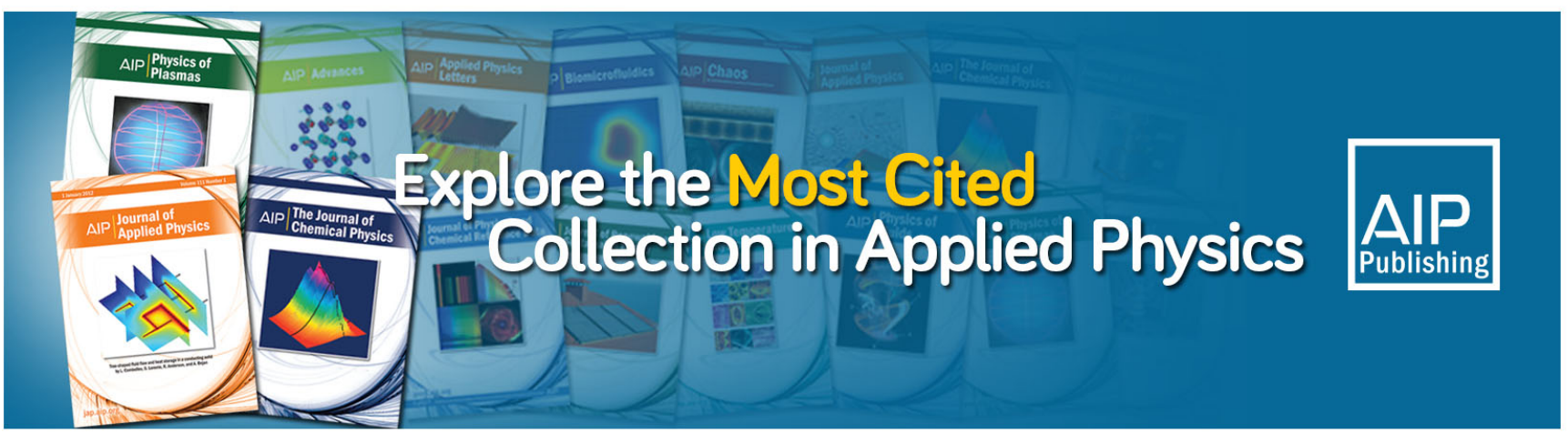




\title{
Intermittent fluctuations in the Alcator C-Mod scrape-off layer ${ }^{a)}$
}

\author{
O. E. Garcia, ${ }^{1, b)}$ S. M. Fritzner, ${ }^{1}$ R. Kube,${ }^{1}$ I. Cziegler, ${ }^{2}$ B. LaBombard ${ }^{3}$ and J. L. Terry ${ }^{3}$ \\ ${ }^{1}$ Department of Physics and Technology, University of Troms $\phi, N-9037$ Troms $\phi$, Norway \\ ${ }^{2}$ Center for Energy Research, University of California, San Diego, California 92093, USA \\ ${ }^{3}$ MIT Plasma Science and Fusion Center, Cambridge, Massachusetts 02139, USA
}

(Received 16 December 2012; accepted 13 February 2013; published online 25 April 2013)

Fluctuations in the boundary region of the Alcator C-Mod tokamak have been analyzed using gas puff imaging data. It is found that the fluctuation amplitudes in the near scrape-off layer follow a normal distribution while the far scrape-off layer fluctuations are dominated by large amplitude bursts due to radial motion of blob-like structures and have a positively skewed and flattened amplitude probability distribution. Conditional averaging of the time series reveals burst wave forms with a fast rise and slow decay and exponentially distributed burst amplitudes and waiting times. Based on this, a stochastic model of the burst dynamics is constructed. The model predicts that fluctuation amplitudes should follow a Gamma distribution and that there is a parabolic relation between the skewness and the kurtosis moments of the fluctuations. This is shown to compare favorably with the gas puff imaging data over a range of line-averaged plasma densities. (C) 2013 AIP Publishing LLC. [http://dx.doi.org/10.1063/1.4802942]

\section{INTRODUCTION}

Cross-field transport of particles and heat in the scrapeoff layer (SOL) of magnetically confined plasmas is dominated by radial motion of blob-like structures. ${ }^{1-5}$ The average radial particle and heat fluxes caused by such filaments depend on their amplitude distribution and frequency of occurrence. ${ }^{6-10}$ The statistical properties of plasma fluctuations in the SOL are thus crucial for development of a firstprinciples description of transport and main-chamber interactions, and may also prove important for understanding the empirical discharge density limit. ${ }^{11-20}$

Fluctuations in Alcator C-Mod SOL have been investigated by analysis of gas puff imaging (GPI) measurements at the outboard mid-plane edge region in Ohmically heated, lower single null discharges. This reveals frequent occurrence of large amplitude bursts with a fast rise and slow decay in the far SOL. Using conditional averaging, the waiting times between these burst events are found to be exponentially distributed. This implies that large amplitude blobs occur randomly and at a constant average rate in the far SOL, and are thus uncorrelated. The average burst duration is found to be independent of the burst amplitude and the line-averaged plasma density.

Based on these statistical properties, a stochastic model for the intermittent SOL plasma fluctuations is constructed, assuming a superposition of bursts occurring in accordance with a Poisson process. ${ }^{20}$ The model input parameters are the burst duration and waiting times and amplitudes. The role that these quantities play in the observations of large plasma densities and fluctuation levels in the far SOL is elucidated. In particular, as a direct consequence of this simple model, the mean plasma density is shown to be proportional to the average burst amplitude and the ratio of the burst

\footnotetext{
a) Paper YI3 1, Bull. Am. Phys. Soc. 57, 370 (2012).

b) Invited speaker. Electronic mail: odd.erik.garcia@uit.no
}

duration and average waiting time. An additional consequence is that there must be a parabolic relation between the skewness and the kurtosis moments, independent of the burst wave form and amplitude distribution. ${ }^{20}$

For exponentially distributed burst amplitudes, this stochastic model reveals that the probability density function (PDF) for the plasma fluctuations is a Gamma distribution with the scale parameter given by the average burst amplitude and the shape parameter by the ratio of the burst duration and waiting times. This Gamma distribution can be rewritten solely in terms of the mean and rms values of the plasma density. Accordingly, it does not involve any free fit parameters when compared to experimental measurements. The PDF changes from a normal distribution for small relative fluctuation levels, typical for the near SOL region, to an exponential distribution for relative fluctuations of order unity, typical for the far SOL region. These predictions of the stochastic model are shown to compare favorably with GPI measurements on Alcator C-Mod. The role of radial motion of blob-like structures for large SOL plasma densities and fluctuation levels, and the dependence on line-averaged particle density is also discussed.

This paper is organized as follows. In Sec. II, we discuss the diagnostics systems on Alcator C-Mod used in this work. Section III presents results from GPI measurements, including probability distributions and conditional averaging. The stochastic model of the SOL plasma fluctuations is described in Sec. IV. Finally, a discussion of the results and our conclusions are presented in Sec. V.

\section{DIAGNOSTICS ON ALCATOR C-MOD}

This paper reports on experiments performed during the FY2010 run campaign using Langmuir probe and GPI data from Alcator C-Mod run 1100803. This comprises a fourpoint scan in the line-averaged density $\bar{n}_{\mathrm{e}}$ with the Greenwald fraction $\bar{n}_{\mathrm{e}} / n_{\mathrm{G}}$ from 0.15 to 0.30 . Here the Greenwald density is given by $n_{\mathrm{G}}=\left(I_{\mathrm{p}} / \pi a^{2}\right) 10^{20} \mathrm{~m}^{-3}$, where $I_{\mathrm{p}}$ is the plasma 
current in units of MA and $a$ is the plasma minor radius in units of meters. For this density scan, $I_{\mathrm{p}}=0.8 \mathrm{MA}$ and $n_{\mathrm{G}}=5.26 \times 10^{20} \mathrm{~m}^{-3}$. A toroidal magnetic field of $4.0 \mathrm{~T}$ was used. These experiments were part of the recent heat flux footprint studies on C-Mod, and have been extensively diagnosed and documented. ${ }^{21}$ The condition at the outer divertor target goes from sheath limited at the lowest density to high recycling at the highest density in this scan.

Fluctuations in the edge region at the outboard midplane were diagnosed using Langmuir probe and GPI measurements. An illustration of the location of these diagnostics in a poloidal cross-section of C-Mod is presented in Fig. 1. A horizontal scanning Langmuir probe with a four-pin probe head is located at $Z=10.88 \mathrm{~cm}$ and is operated in a voltage sweep mode with a sampling rate of $5 \mathrm{MHz}$. Data reported here come from all four Langmuir probes on the scanning probe head. Measurements of local particle density and temperature from each probe are made every $0.3 \mathrm{~ms}$ during the probe trajectory, corresponding to roughly $0.3 \mathrm{~mm}$ of probe travel. The resulting particle density and temperature profiles which consist of approximately 400 data points are then fitted by a smooth spline curve. The scanning probe trajectory starts from the location $(R, Z)=(98.00,10.88) \mathrm{cm}$, and moves along the negative $R$ direction. Further information about the Langmuir probe diagnostic on Alcator C-Mod can be found in Ref. 11.

The GPI diagnostic consists of a $9 \times 10$ array of invessel optical fibres with toroidally viewing, horizontal lines of sight. The plasma emission collected in the views is filtered for He I $(587 \mathrm{~nm})$ line emission that is locally enhanced

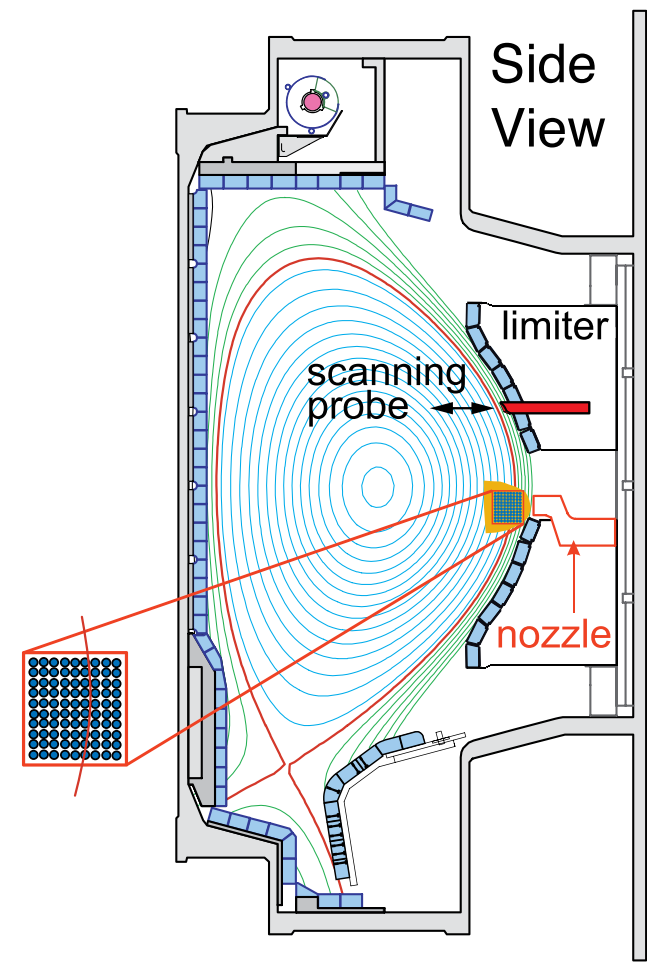

FIG. 1. Cross-section of Alcator C-Mod showing the location of the horizontal scanning probe and the gas puff imaging diagnostic at the outboard midplane region. In the enlargement of the $9 \times 10$ diode array, the vertical line shows a typical location of the last closed magnetic flux surface. in the object plane by an extended He gas puff from a nearby nozzle. The fibres are coupled to high sensitivity avalanche photo diodes and the signals are digitized at a rate of $2 \times 10^{6}$ frames per second. The viewing area covers the major radius from 88.00 to $91.08 \mathrm{~cm}$ and vertical coordinate from -4.51 to $-1.08 \mathrm{~cm}$ with an in-focus spot size of $3.8 \mathrm{~mm}$ for each of the 90 individual channels. The radial position of the last closed flux surface at the vertical centre of the image, $Z=-2.61 \mathrm{~cm}$, is in the range from 89.4 to $89.7 \mathrm{~cm}$ for all the discharges presented here. The limiter radius mapped to this vertical position is at $R=91.0 \mathrm{~cm}$. For each discharge, the GPI diagnostic yields $0.25 \mathrm{~s}$ usable data time series during the flat-top of the plasma current. By combining data from two discharges at the same $\bar{n}_{\mathrm{e}}$ and two nearby diode channels at the same radial position with identical statistical properties, we obtain time series of one second duration which allows calculation of statistical averages with high accuracy. Further information about this GPI diagnostic on Alcator C-Mod can be found in Ref. 22.

Excitation of the neutral He gas, and thus the intensity of the GPI signals, is determined by the neutral He particle density and a combination of the local electron particle density and temperature. ${ }^{23,24}$ Inside the last closed magnetic flux surface, the dense and hot background plasma ionizes most of the injected He gas which results in low light emission levels. In the limiter shadow region, the thin and cold ambient plasma is unable to cause significant emission levels from the neutral He gas except in the presence of large-amplitude blob structures. For these reasons, the radial variation of the average GPI intensity differs from that of the electron particle density. However, Langmuir probe measurements have demonstrated that the electron particle density and temperature fluctuate in phase. ${ }^{14,25}$ In the following, we will therefore assume that the fluctuating part of the plasma density may be approximated by fluctuations in the GPI intensity signals from Alcator C-Mod. The following analysis of GPI data elucidates the statistical properties of these fluctuations.

\section{EXPERIMENTAL MEASUREMENTS}

In Fig. 2, we present the radial profile of the timeaveraged particle density obtained from measurements using the horizontal scanning probe on C-Mod. At the vertical position of the probe, the last closed flux surface is estimated to be located between $R=85.9$ and $86.1 \mathrm{~cm}$. In Fig. 2 and following figures, the location of the last closed magnetic flux surface is indicated by a gray shaded region. The particle density profile exhibits a well-known two-layer structure. ${ }^{11-15}$ Close to the separatrix, in the so-called near SOL region, it has a steep profile and moderate fluctuation levels. Beyond this region, in the so-called far SOL, the profile has a roughly exponential decay with much longer scale length and a fluctuation level of order unity. As the discharge density limit is approached, the far SOL profile becomes broader and the break point moves radially inwards such that the far SOL profile effectively extends all the way to the magnetic separatrix or even inside it. ${ }^{26}$

As an illustration of the plasma dynamics in the SOL we present in Fig. 3 the GPI signals at three different radial 


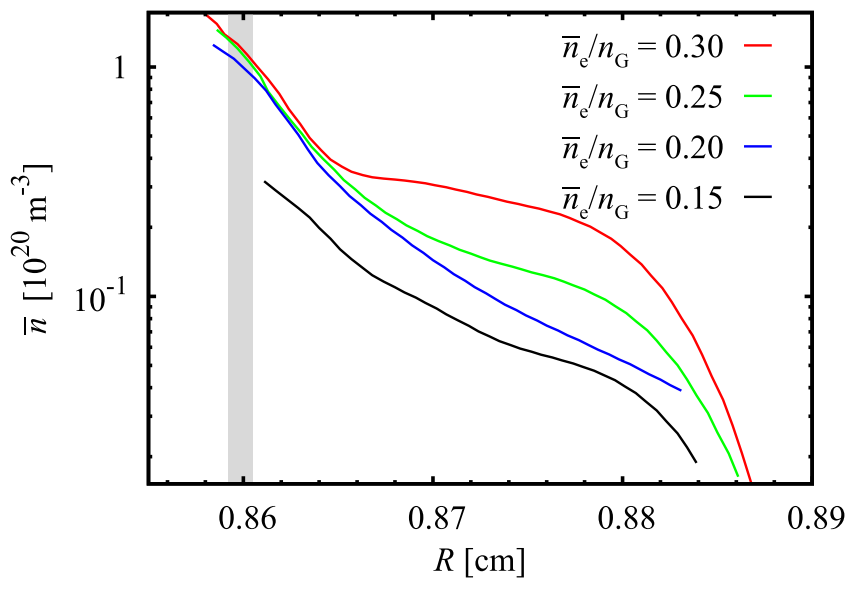

FIG. 2. Radial profile of the particle density measured by the horizontal scanning probe for four different line-averaged densities as function of major radius for $Z=10.88 \mathrm{~cm}$.

positions for $Z=-2.61 \mathrm{~cm}$ and $\bar{n}_{\mathrm{e}} / n_{\mathrm{G}}=0.15$. The innermost diode view position shown here is in the vicinity of the last closed magnetic flux surface and the outermost position is in the limiter shadow where the magnetic field lines intersect limiter structures and have relatively short connection lengths $(\sim 1 \mathrm{~m})$. It is clearly seen that time series from single point measurements in the SOL are dominated by large amplitude bursts. These bursts are associated with radial motion of blob-like structures, which have a steep front and a trailing wake in the spatial domain. Due to the low emission levels in the limiter shadow region, bursts appear on top of an essentially zero-emission baseline as is clearly seen in Fig. 3 at the location $R=91.08 \mathrm{~cm}$.

In Fig. 4, we show the radial variation of the relative fluctuation level of the GPI signals. The fluctuations increase drastically in magnitude with radial distance into the SOL. As shown in Fig. 3, the raw time series are here dominated by large-amplitude bursts due to the radial motion of bloblike structures. This results in positively skewed and flattened PDFs of the GPI signals. This is clear from the radial profile of the skewness and kurtosis moments of the GPI signals presented in Figs. 5 and 6. Inside the last closed

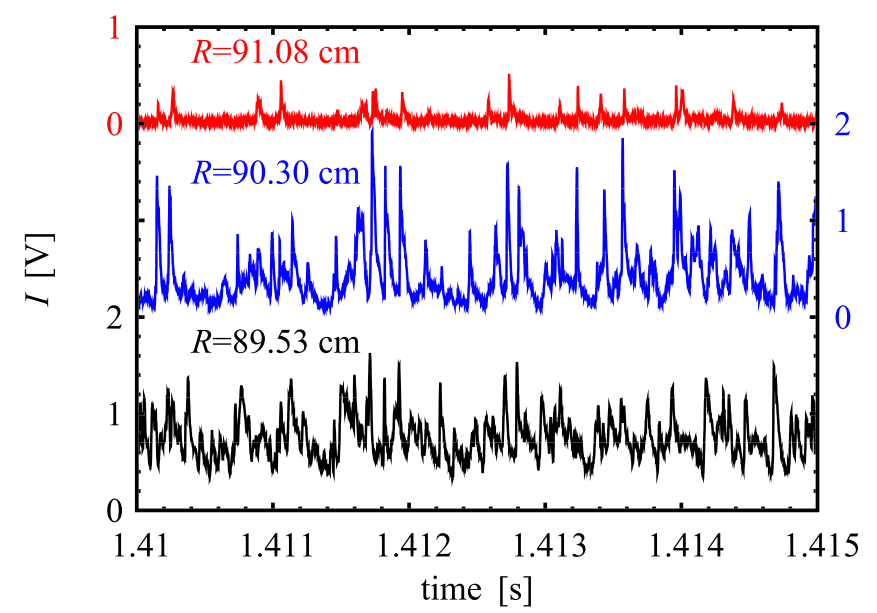

FIG. 3. Time series of the raw GPI signals (in volts) for $\bar{n}_{\mathrm{e}} / n_{\mathrm{G}}=0.15$ at $Z=-2.61 \mathrm{~cm}$ and various radial positions in the SOL.

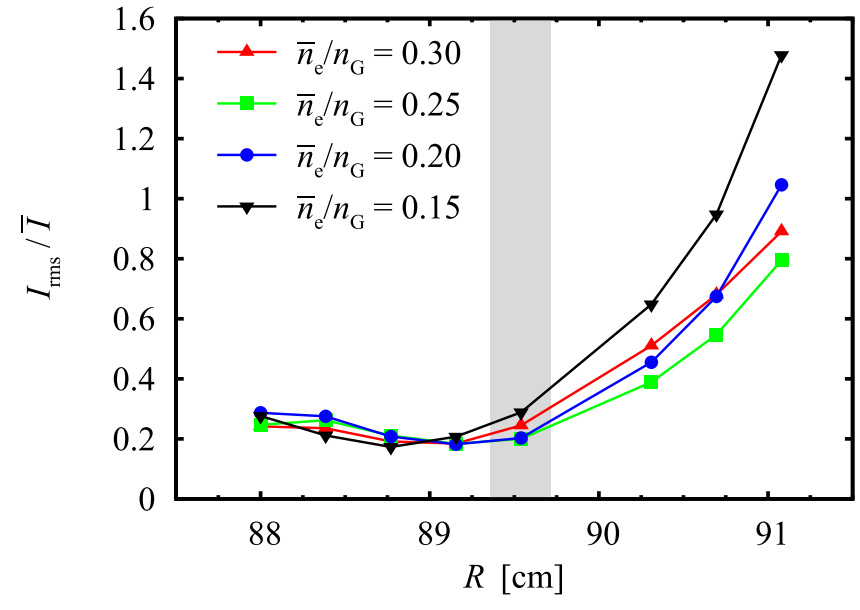

FIG. 4. Radial variation of relative GPI signal fluctuation level.

magnetic flux surface, the skewness and excess kurtosis are small, indicating nearly normal distributed fluctuation amplitudes here. This is consistent with the nearly symmetric fluctuations seen in Fig. 3 for $R=89.53 \mathrm{~cm}$ and $\bar{n}_{\mathrm{e}} / n_{\mathrm{G}}=0.15$.

The PDF of the GPI signals is presented in Fig. 7 for $\bar{n}_{\mathrm{e}} / n_{\mathrm{G}}=0.20$ and the four GPI fibre view positions radially outside the separatrix. Note that the PDFs for the experimental data comprise more than four decades on the ordinate axis - a consequence of the long data time series used in this analysis. It is clearly seen that the PDF changes from a normal distribution in the near SOL region to positively skewed and flattened in the far SOL. In the limiter shadow, the PDF has an exponential tail towards large signal amplitudes. This change in the shape of the PDF in the SOL is qualitatively similar to what has previously been found from Langmuir probe measurements in several tokamak experiments. ${ }^{11,14,15,27,28}$

The radial motion of blob-like structures through the SOL results in single-point recordings of the plasma density dominated by bursts with a fast rise and a slow decay. This is clearly seen in Fig. 3 and further demonstrated by the asymmetric wave form obtained from conditional averaging presented in Fig. 8 for $R=90.69 \mathrm{~cm}$. The amplitude condition

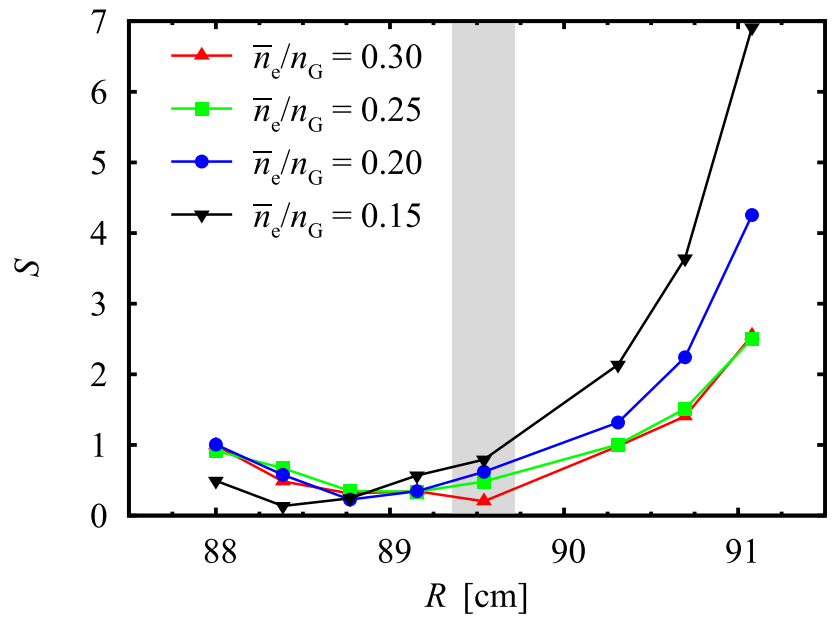

FIG. 5. Radial variation of the skewness of GPI signal fluctuations. 


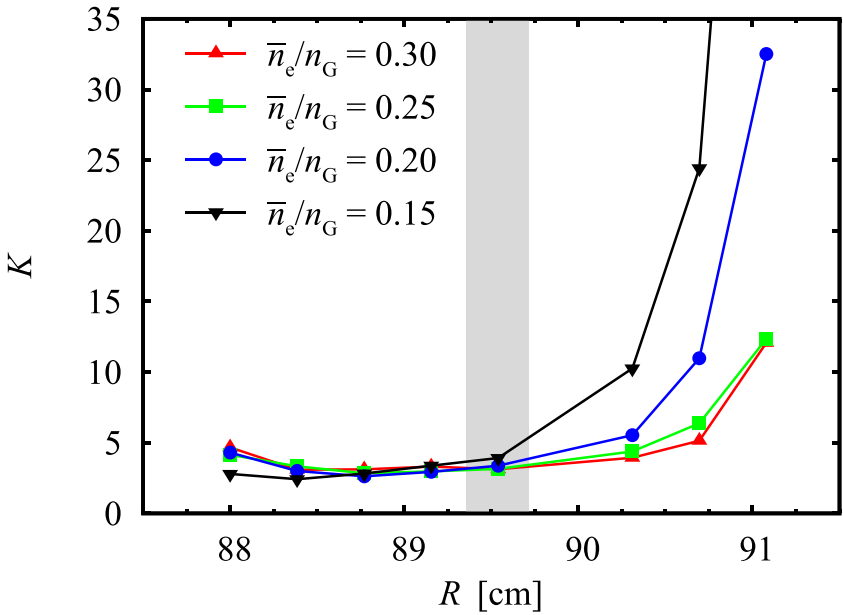

FIG. 6. Radial variation of the kurtosis of GPI signal fluctuations.

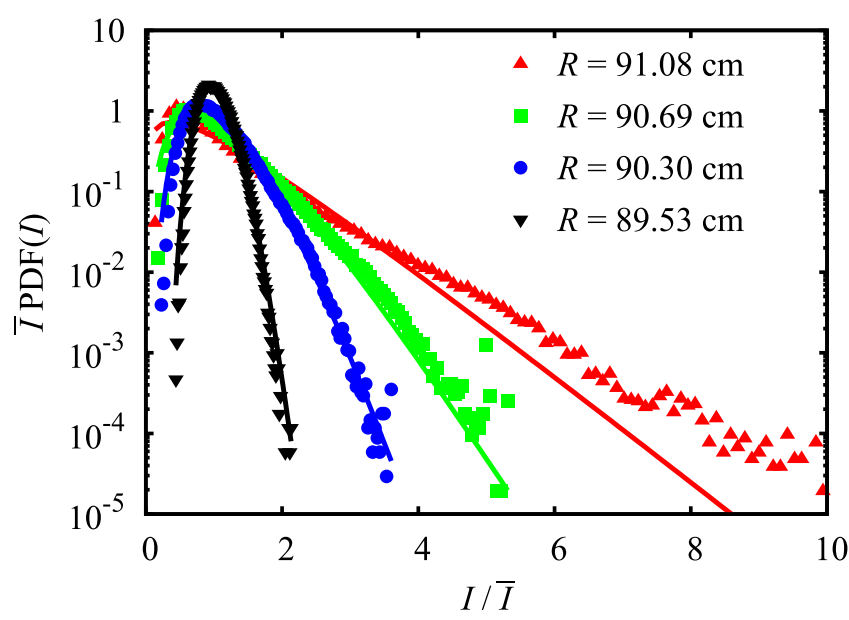

FIG. 7. PDF of GPI intensity signals for $\bar{n}_{\mathrm{e}} / n_{\mathrm{G}}=0.25$ and various radial positions in the SOL. The full lines are predictions of the stochastic model.

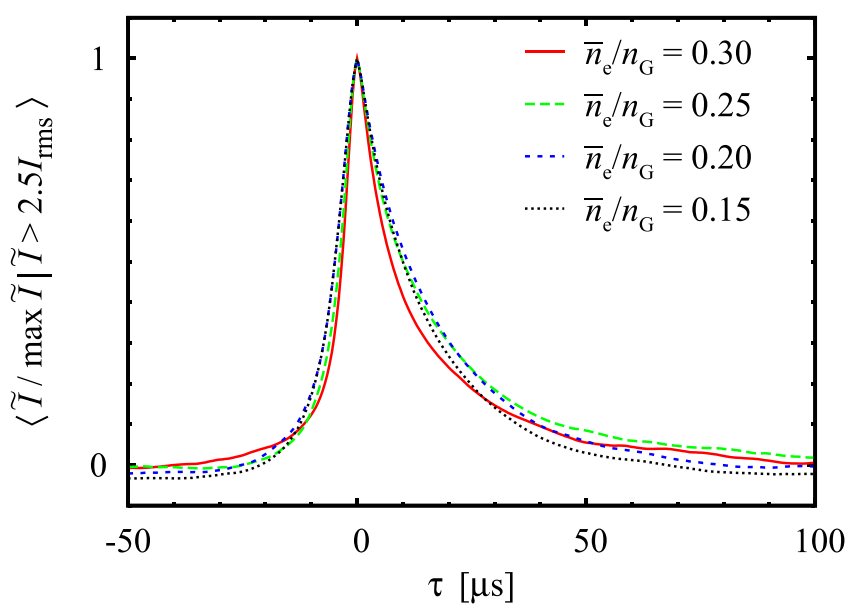

FIG. 8. Conditionally averaged wave form for GPI signal burst amplitudes larger than 2.5 times the standard deviation at $R=90.69 \mathrm{~cm}$ as function of time lag from peak amplitude.

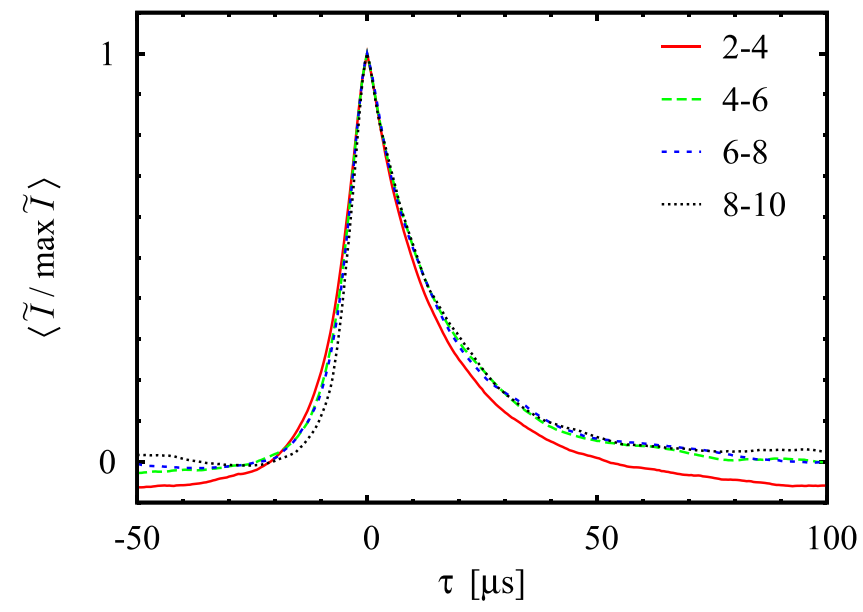

FIG. 9. Conditionally averaged wave form for GPI signal burst amplitudes at $R=90.69 \mathrm{~cm}$ for $\bar{n}_{\mathrm{e}} / n_{\mathrm{G}}=0.15$ and amplitude thresholds in factors of $\max \tilde{I} / I_{\mathrm{rms}}$ given by the range stated in the legend.

used was max $\tilde{I}>2.5 I_{\mathrm{rms}}$, which resulted in several thousand burst events for each line-averaged density due to the long time series available. The typical amplitude is nearly 4 times the rms value and the duration is approximately $25 \mu$ s for all line-averaged densities. It is further seen that the average burst duration is the same for all line-averaged densities. This is again similar to what has previously been found from probe measurements. ${ }^{14-16}$

By requiring that the GPI signal burst amplitudes are within a specified interval, we are able to elucidate the amplitude dependence of the burst duration. In Fig. 9, we present the conditionally averaged GPI signals for $\bar{n}_{\mathrm{e}} / n_{\mathrm{G}}=0.15$ at $R=90.69 \mathrm{~cm}$ (note that this is normalized to the peak amplitude of the wave form) for large amplitude events in the ranges $2-4,4-6,6-8$, and $8-10$ times the root mean square value at this position. In all cases, the burst wave form has the familiar shape with a fast rise and a slow decay. After normalization with the peak amplitude for each condition interval, the average burst duration is found to be independent of burst amplitude. Similar results are found for all lineaveraged densities.

The waiting time between large amplitude bursts is also obtained from the conditional averaging. As shown in Fig. 10, the burst waiting times are found to be exponentially distributed for all line-averaged densities. ${ }^{29}$ An exponential distribution describes the time between events for a Poisson process, in which events occur randomly and at a constant average rate. This implies that large-amplitude blobs appearing in the far SOL are uncorrelated. It is also found that the peak burst amplitudes in the far SOL are exponentially distributed, in accordance with the exponential tail for large amplitudes seen in Fig. 7.

\section{STOCHASTIC MODELLING}

The experimental measurements presented here suggest that the plasma density in the SOL can be represented as a superposition of uncorrelated pulses ${ }^{20}$

$$
\Phi(t)=\sum_{k} A_{k} \psi\left(t-t_{k}\right),
$$




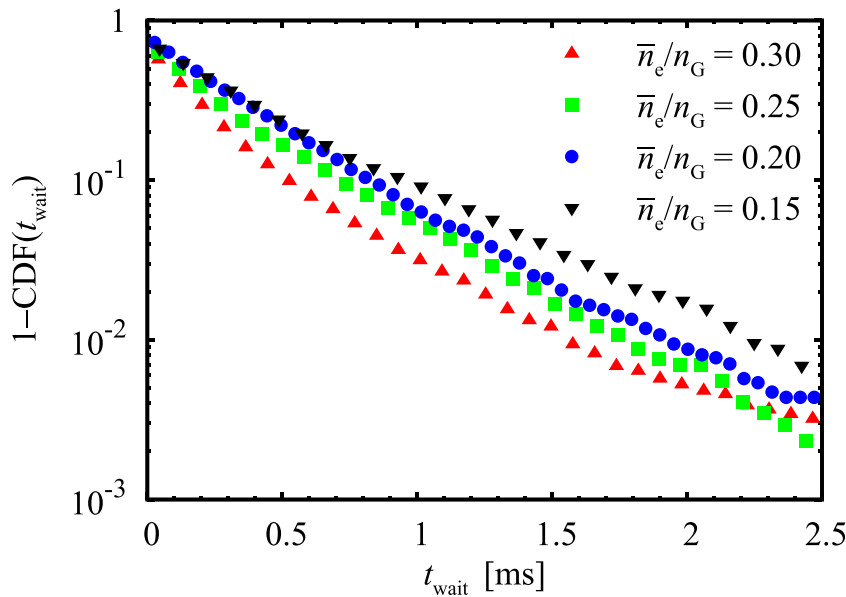

FIG. 10. Complementary cumulative distribution function of waiting times between burst events with maximum fluctuation amplitude larger than 2.5 times the standard deviation value at $R=90.69 \mathrm{~cm}$.

where $t_{k}$ is the burst arrival time and $A_{k}$ the burst amplitude for event $k$. The burst wave form $\psi(t)$ is assumed to be a double exponential

$$
\Psi(t)= \begin{cases}\exp \left(\frac{t}{\tau_{\mathrm{r}}}\right) & \text { for } t \leq 0 \\ \exp \left(-\frac{t}{\tau_{\mathrm{d}}-\tau_{\mathrm{r}}}\right) & \text { for } t>0\end{cases}
$$

with a constant burst rise time $\tau_{\mathrm{r}}$ and duration $\tau_{\mathrm{d}}$, in agreement with the experimental results presented in Figs. 8 and 9. The bursts are assumed to occur in accordance to a Poisson process, from which it follows that the burst waiting times, given by $\tau_{k}=t_{k}-t_{k-1}$, are exponentially distributed. This is consistent with the experimental results presented in Fig. 10. The burst amplitudes are also taken to be exponentially distributed

$$
P_{A}(A)=\frac{1}{\langle A\rangle} \exp \left(-\frac{A}{\langle A\rangle}\right)
$$

where $\langle A\rangle$ is the average burst amplitude. The mean value of the signal in Eq. (1) is given by ${ }^{20}$

$$
\langle\Phi\rangle=\frac{\tau_{\mathrm{d}}}{\tau_{\mathrm{w}}}\langle A\rangle,
$$

where $\tau_{\mathrm{w}}$ is the average burst waiting time. This equation elucidates the role of burst duration and waiting times and amplitudes for large SOL plasma densities.

The relative fluctuation level can be written as $\Phi_{\mathrm{rms}} /\langle\Phi\rangle=\left(\tau_{\mathrm{w}} / \tau_{\mathrm{d}}\right)^{1 / 2}$, while the skewness and kurtosis moments for $\Phi$ are given by ${ }^{20}$

$$
S=\left(\frac{4 \tau_{\mathrm{w}}}{\tau_{\mathrm{d}}}\right)^{1 / 2}, \quad K=3+\frac{6 \tau_{\mathrm{w}}}{\tau_{\mathrm{d}}}
$$

Like the relative fluctuation level, also the skewness and kurtosis increase with the ratio $\tau_{\mathrm{w}} / \tau_{\mathrm{d}}$. The parameter $\gamma=\tau_{\mathrm{d}} / \tau_{\mathrm{w}}$ is thus a measure of intermittency in the signal given by Eq. (1). From Eq. (5), it follows that there is a parabolic

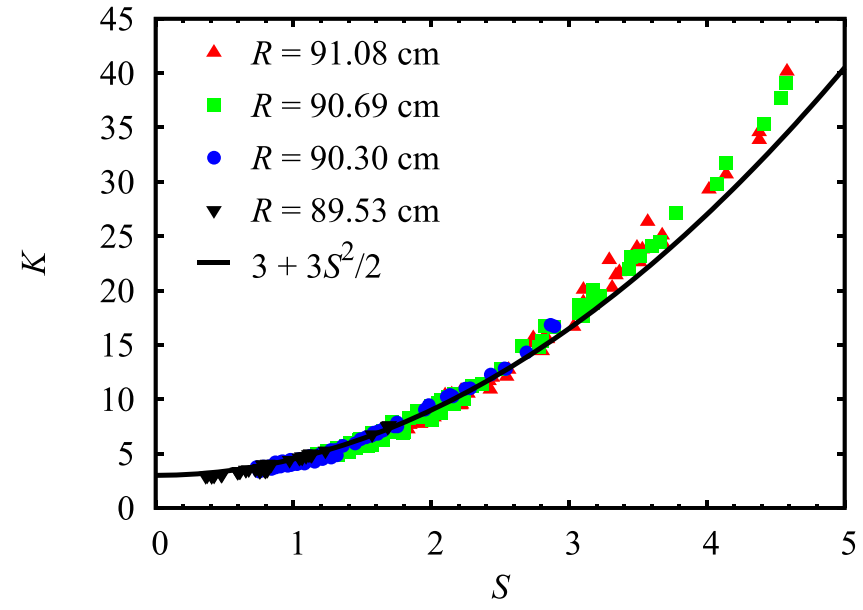

FIG. 11. Kurtosis versus skewness for all GPI diode view positions in the SOL and all 8 discharges in the density scan. The full line is the prediction of the stochastic model.

relation between these moments given by $K=3+3 S^{2} / 2$. In Fig. 11, we present the kurtosis as function of skewness calculated for all GPI fibre view positions located in the SOL for all 8 discharges used in the present density scan. It is again seen that the skewness and kurtosis moments increase radially outwards in the SOL. Also shown in Fig. 11 is the predicted parabolic relation given by Eq. (5). This is an excellent description of the experimental data for all but the outermost GPI diode view positions. This apparent discrepancy is due to reduced emission from the neutral He gas for the thin and cold plasma in the limiter shadow region, as discussed in Sec. II. Here significant emission levels only arise in the presence of large amplitude blob structures, as seen in Fig. 3. This leads to excessively large higher order moments of the fluctuations that is not captured by the stochastic model. This is evidently the cause of the deviation from a parabolic relation between the skewness and the kurtosis moments for the signals from the outermost GPI views positions in Fig. 11. It is noted that such a parabolic relation has previously been found for several magnetized plasma experiments. $^{27,28,30,31}$

For an exponential wave form and exponentially distributed burst amplitudes, the PDF for the signal given by the model (1) can be shown to be a Gamma distribution ${ }^{20}$

$$
\langle\Phi\rangle P_{\Phi}(\Phi)=\frac{\gamma}{\Gamma(\gamma)}\left(\frac{\gamma \Phi}{\langle\Phi\rangle}\right)^{\gamma-1} \exp \left(-\frac{\gamma \Phi}{\langle\Phi\rangle}\right),
$$

where the scale parameter is given by $\langle\Phi\rangle / \gamma$ and the shape parameter is $\gamma=\langle\Phi\rangle^{2} / \Phi^{2}$ rms. It should be noted that there are no free fit parameters when comparing this distribution to experimental measurements. In Fig. 7 we present the corresponding Gamma distribution, which is seen to be an excellent fit to the data for all but the outermost GPI diode view position. In the limiter shadow the PDF of the measured signals has an elevated tail relative to the Gamma distribution, which is likely due to suppressed emission in the thin and cold far SOL plasma as discussed above. The Gamma distribution given by Eq. (6) has previously been found to accurately describe the ion saturation current signal 
measured by Langmuir probes in the SOL of Tokamak á configuration variable across a broad range of plasma parameters. $^{27,28}$

\section{DISCUSSION AND CONCLUSIONS}

GPI measurements in the SOL of Alcator C-Mod have shown that plasma fluctuations are dominated by large amplitude bursts due to radial motion of blob-like structures. The burst wave form is asymmetric with a fast rise and slow decay, and a duration that is independent of burst amplitude and line-averaged density. Conditional averaging reveals that the burst waiting times and amplitudes are exponentially distributed, thus blobs appearing in the far SOL are uncorrelated. The PDF of the GPI signals changes from a normal distribution in the near SOL to positively skewed and flattened with an exponential tail in the far SOL.

A stochastic model of burst dynamics is constructed based on three parameters: burst duration, waiting time and amplitude. Consistent with the predictions of the model, the PDFs of the GPI intensity fluctuations are well described by a Gamma distribution and there is correspondingly a parabolic relation between the skewness and the flatness moments. These results indicate that the stochastic model is an accurate description of the burst dynamics in the tokamak SOL for the C-Mod plasmas investigated here.

According to the stochastic model, the relative fluctuation level is given by the degree of burst overlap, $\Phi_{\mathrm{rms}} /\langle\Phi\rangle=\left(\tau_{\mathrm{w}} / \tau_{\mathrm{d}}\right)^{1 / 2}$. As seen in Fig. 4, the relative GPI fluctuation level increases radially outwards in the SOL. This suggests that there is a change in the ratio between the burst duration and the waiting time, with strong overlap of burst events in the edge and near SOL region. The model explains the observed change in the PDF from a normal distribution in the near SOL to positively skewed and flattened in the far SOL. It should be noted that in the case of strong burst overlap, the stochastic model predicts a normal distribution. This follows from the central limit theorem and is independent of the burst wave form and amplitude distribution. ${ }^{20}$

The average plasma density is proportional to the average burst amplitude and the ratio of the burst duration and waiting times. There are two means by which the average burst amplitude increases with the Greenwald density fraction. First, as the plasma density increases, the blob structures are formed in regions with larger plasma density and thus have larger initial amplitudes. Second, the radial velocity of the blob structures increases with the ratio $\bar{n}_{\mathrm{e}} / n_{\mathrm{G}} \cdot{ }^{15,32,33}$ This results in shorter radial transit times and thereby less parallel losses along the magnetic field. This also leads to larger blob amplitudes and radial particle fluxes in the far SOL.

In summary, we have presented new experimental measurements demonstrating that plasma fluctuations in the far SOL are dominated by large amplitude bursts with an asymmetric wave form and an exponential distribution of waiting times. The latter implies that blob structures in the SOL are uncorrelated. Based on this, a stochastic model has been constructed which predicts Gamma distributed plasma fluctuation amplitudes and a parabolic relation between the skewness and the kurtosis moments. This is shown to compare favorably with GPI experiments for a range of lineaveraged particle densities in Alcator C-Mod plasmas.

${ }^{1}$ D. A. D’Ippolito, J. R. Myra, and S. J. Zweben, Phys. Plasmas 18, 060501 (2011).

${ }^{2}$ O. E. Garcia, Plasma Fusion Res. 4, 019 (2009).

${ }^{3}$ S. I. Krasheninnikov, D. A. D'Ippolito, and J. R. Myra, J. Plasma Phys. 74, 679 (2008)

${ }^{4}$ S. J. Zweben, J. A. Boedo, O. Grulke, C. Hidalgo, B. LaBombard, R. J. Maqueda, P. Scarin, and J. L. Terry, Plasma Phys. Controlled Fusion 49, S1 (2007).

${ }^{5}$ D. A. D'Ippolito, J. R. Myra, and S. I. Krasheninnikov, Contrib. Plasma Phys. 44, 205 (2004).

${ }^{6}$ D. A. D'Ippolito, J. R. Myra, and S. I. Krasheninnikov, Phys. Plasmas 9, 222 (2002)

${ }^{7}$ O. E. Garcia, N. H. Bian, and W. Fundamenski, Phys. Plasmas 13, 082309 (2006); 12, 090701 (2005).

${ }^{8}$ R. Kube and O. E. Garcia, Phys. Plasmas 18, 102314 (2011); 19, 042305 (2012).

${ }^{9}$ B. Lipschultz, X. Bonnin, G. Counsell, A. Kallenbach, A. Kukushkin, K. Krieger, A. Leonard, A. Loarte, R. Neu, R. A. Pitts, T. Rognlien, J. Roth, C. Skinner, J. L. Terry, E. Tsitrone, D. Whyte, S. Zweben, N. Asakura, D. Coster, R. Doerner, R. Dux, G. Federici, M. Fenstermacher, W. Fundamenski, P. Ghendrih, A. Herrmann, J. Hu, S. Krasheninnikov, G. Kirnev, A. Kreter, V. Kurnaev, B. LaBombard, S. Lisgo, T. Nakano, N. Ohno, H. D. Pacher, J. Paley, Y. Pan, G. Pautasso, V. Philipps, V. Rohde, D. Rudakov, P. Stangeby, S. Takamura, T. Tanabe, Y. Yang, and S. Zhu, Nucl. Fusion 47, 1189 (2007).

${ }^{10}$ B. A. Carreras, J. Nucl. Mater. 337-339, 315 (2005).

${ }^{11}$ B. LaBombard, R. L. Boivin, M. Greenwald, J. Hughes, B. Lipschultz, D. Mossessian, C. S. Pitcher, J. L. Terry, S. J. Zweben, and Alcator Group, Phys. Plasmas 8, 2107 (2001); B. LaBombard, J. W. Hughes, D. Mossessian, M. Greenwald, B. Lipschultz, J. L. Terry, and Alcator C-Mod Team, Nucl. Fusion 45, 1658 (2005).

${ }^{12}$ B. Lipschultz, B. LaBombard, C. S. Pitcher, and R. Boivin, Plasma Phys. Controlled Fusion 44, 733 (2002).

${ }^{13}$ D. G. Whyte, B. L. Lipschultz, P. C. Stangeby, J. Boedo, D. L. Rudakov, J. G. Watkins, and W. P. West, Plasma Phys. Controlled Fusion 47, 1579 (2005).

${ }^{14}$ D. L. Rudakov, J. A. Boedo, R. A. Moyer, P. C. Stangeby, J. G. Watkins, D. G. Whyte, L. Zeng, N. H. Brooks, R. P. Doerner, T. E. Evans, M. E. Fenstermacher, M. Groth, E. M. Hollmann, S. I. Krasheninnikov, C. J. Lasnier, A. W. Leonard, M. A. Mahdavi, G. R. McKee, A. G. McLean, A. Yu. Pigarov, W. R. Wampler, G. Wang, W. P. West, and C. P. C. Wong, Nucl. Fusion 45, 1589 (2005); J. A. Boedo, D. Rudakov, R. Moyer, G. R. McKee, R. J. Colchin, M. J. Schaffer, P. G. Stangeby, W. P. West, S. L. Allen, T. E. Evans, R. J. Fonck, E. M. Hollmann, S. Krasheninnikov, A. W. Leonard, W. Nevins, M. A. Mahdavi, G. D. Porter, G. R. Tynan, D. G. Whyte, and X. Xu, Phys. Plasmas 10, 1670 (2003).

${ }^{15}$ O. E. Garcia, J. Horacek, R. A. Pitts, A. H. Nielsen, W. Fundamenski, V. Naulin, and J. Juul Rasmussen, Nucl. Fusion 47, 667 (2007); J. Nucl. Mater. 363-365, 575 (2007); Plasma Phys. Controlled Fusion 49, B47 (2007).

${ }^{16}$ G. Y. Antar, G. Counsell, Y. Yu, B. LaBombard, and P. Devynck, Phys. Plasmas 10, 419 (2003).

${ }^{17}$ D. A. D'Ippolito and J. R. Myra, Phys. Plasmas 13, 062503 (2006)

${ }^{18}$ P. N. Guzdar, R. G. Kleva, P. K. Kaw, R. Singh, B. LaBombard, and M. Greenwald, Phys. Plasmas 14, 020701 (2007).

${ }^{19}$ X. Q. Xu, W. M. Nevins, T. D. Rognlien, R. H. Bulmer, M. Greenwald, A. Mahdavi, L. D. Pearlstein, and P. Snyder, Phys. Plasmas 10, 1773 (2003).

${ }^{20}$ O. E. Garcia, Phys. Rev. Lett. 108, 265001 (2012); O. E. Garcia, I. Cziegler, R. Kube, B. LaBombard, and J. L. Terry, J. Nucl. Mater. (to be published).

${ }^{21}$ B. LaBombard, J. L. Terry, J. W. Hughes, D. Brunner, J. Payne, M. L. Reinke, I. Cziegler, R. Granetz, M. Greenwald, I. H. Hutchinson, J. Irby, Y. Lin, B. Lipschultz, Y. Ma, E. S. Marmar, W. L. Rowan, N. Tsujii, G. Wallace, D. G. Whyte, S. Wolfe, S. Wukitch, G. Wurden, and Alcator C-Mod Team, Phys. Plasmas 18, 056104 (2011).

${ }^{22}$ I. Cziegler, J. L. Terry, J. W. Hughes, and B. LaBombard, Phys. Plasmas 17, 056120 (2010).

${ }^{23}$ D. P. Stotler, B. LaBombard, J. L. Terry, and S. J. Zweben, J. Nucl. Mater. 313-316, 1066 (2003). 
${ }^{24}$ D. Russell, J. R. Myra, D. A. D’Ippolito, T. L. Munsat, Y. Sechrest, R. J. Maqueda, D. P. Stotler, S. J. Zweben, and NSTX Team, Phys. Plasmas 18, 022306 (2011).

${ }^{25}$ B. LaBombard, D. Brunner, O. E. Garcia, M. Greenwald, J. W. Hughes, R. Kube, J. L. Terry, and S. J. Zweben, "Mirror Langmuir probe measurements of fluctuation-induced heat and particle transport in the Alcator CMod boundary plasma" in Bulletin of the American Physical Society, 54th Annual Meeting of the Division of Plasma Physics Meeting, BO7-14, Salt Lake City, Utah, October 29-November 2, 2012.

${ }^{26}$ J. L. Terry, N. P. Basse, I. Cziegler, M. Greenwald, O. Grulke, B. LaBombard, S. J. Zweben, E. M. Edlund, J. W. Hughes, L. Lin, Y. Lin, M. Porkolab, M. Sampsell, B. Veto, and S. J. Wukitch, Nucl. Fusion 45, 1321 (2005).

${ }^{27}$ J. P. Graves, J. Horacek, R. A. Pitts, and K. I. Hopcraft, Plasma Phys. Controlled Fusion 47, L1 (2005).

${ }^{28}$ J. Horacek, R. A. Pitts, and J. P. Graves, Czech J. Phys. 55, 271 (2005).

${ }^{29}$ For every real number $x$, the cumulative distribution function (CDF) of a real-valued random variable $X$ is given by $F_{X}(x)=\operatorname{Pr}(X \leq x)$ where
$\operatorname{Pr}(X \leq x)$ is the probability that the random variable $X$ takes on a value less than or equal to $x$. The CDF of a continuous random variable $X$ can be defined in terms of its probability density function $\mathrm{P}_{X}$ as $F_{X}(x)$ $=\int_{-\infty}^{x} d x^{\prime} P_{X}\left(x^{\prime}\right)$. The complementary cumulative distribution function is defined as $\operatorname{Pr}(X>x)=1-F_{X}(x)$. For an exponential distribution $P_{X}=\exp (-X /\langle X\rangle) /\langle X\rangle$ and the complementary cumulative distribution is $1-F_{X}(X)=\exp (-X /\langle X\rangle)$.

${ }^{30}$ F. Sattin, M. Agostini, P. Scarin, N. Vianello, R. Cavazzana, L. Marrelli, G. Serianni, S. J. Zweben, R. J. Maqueda, Y. Yagi, H. Sakakita, H. Koguchi, S. Kiyama, Y. Hirano, and J. L. Terry, Plasma Phys. Controlled Fusion 51, 055013 (2009).

${ }^{31}$ B. Labit, I. Furno, A. Fasoli, A. Diallo, S. H. Müller, G. Plyushchev, M. Podestà, and F. M. Poli, Phys. Rev. Lett. 98, 255002 (2007).

${ }^{32}$ M. Agostini, J. L. Terry, P. Scarin, and S. J. Zweben, Nucl. Fusion 51, 053020 (2011).

${ }^{33}$ R. Kube, O. E. Garcia, B. LaBombard, J. L. Terry, and S. J. Zweben, J. Nucl. Mater. (to be published). 